\title{
A survey of NHS Direct callers' use of health services and the interventions they received
}

Geraldine Byrne School of Nursing and Midwifery, University of Hertfordshire, Hatfield, UK, Janice Morgan and Sally Kendall Centre for Research in Primary and Community Care, University of Hertfordshire, Hatfield, UK and Debbie Saberi NHS Direct, Bedford, UK

\begin{abstract}
National Health Service (NHS) Direct is a 24-h patient-led telephone advice service, based in England and Wales, which aims to help callers self-manage problems and reduce unnecessary demands on other NHS provision. Despite its rapid expansion as a source of health information and advice, little empirical work has investigated its impact and effectiveness. This paper reports on a study that aimed to evaluate the contribution of NHS Direct to the quality of care and its impact on health services within one part of the United Kingdom (UK). A postal survey of callers who had contacted NHS Direct between June 2004 and January 2005 with abdominal pain or cough and/or sore throat was conducted. Of 268 callers, over $90 \%$ reported they had followed the advice given by NHS Direct to self-care or to contact another health service. Fifty-one of the callers were referred to an Accident and Emergency department and, of these, 39 $(76 \%)$ received a prescription and $20(39 \%)$ were admitted to hospital. One hundred and forty-four callers were referred to a General Practitioner (GP) and, of these, 88 $(61 \%)$ received a prescription and $21(15 \%)$ were admitted to hospital. Of the 69 callers advised to self-care, $47(68 \%)$ reported that they did not receive any further intervention while $18(26 \%)$ reported they had subsequently contacted a GP and been given a prescription for medication. The paper concludes that most referrals made by NHS Direct to another health service were justified by the interventions received by patients. However a significant minority $(26 \%)$ of callers referred to GPs did not receive any further intervention and there was some duplication of service use. Health professionals need to be aware that patients might have previously contacted another health service and provide advice on effective use of services.
\end{abstract}

Keywords: Accident and Emergency; NHS Direct; nurses' role; primary care; quality of care; telephone advice.

Received: February 2006; accepted: November 2006

\section{Background}

NHS Direct is a 24-h patient-led telephone advice service, based in England and Wales, which aims to help callers to self-manage problems and reduce unnecessary demands on other National Health Service (NHS) provision (Munro et al., 2000a).

Address for correspondence: Dr Geraldine Byrne, School of Nursing and Midwifery, Wright Building, University of Hertfordshire, College Lane, Hatfield, Herts, AL10 9AB. Email: G.1.Byrne@herts.ac.uk
NHS Direct is the world's largest health care telephone advice service (Munro, 2002) and is leading the current international trend towards telemedicine and telephone consultation (Hanlon et al., 2005). Telephone triage and telephone consultation are now used in many countries; their development being partly driven by increasing demand for General Practitioner (GP) and Accident and Emergency (A\&E) services (Christensen and Olensen, 1998; Lattimer et al., 1998; Sprivulis et al., 2004). A systematic review of the effects of telephone consultation and triage (Bunn et al., 2005) 
found telephone consultation can reduce GP visits and do not appear to increase adverse effects, but the evidence base is limited.

In the United Kingdom (UK), NHS Direct is central to the Department of Health (DOH) policy of improving access to health care, enhancing patients' experiences and promoting empowerment and choice among the users of health services (DOH, 2002). Staffed by nurses, it reflects the move towards extending nurses' roles to provide a quick and responsive health care system and increasing the flexibility of primary care provision. Despite its rapid expansion as a source of health information and advice, there is little empirical work that has investigated the impact and effectiveness of NHS Direct. Most of the literature is commentary and professional debate about its value, much of which reveals medical staff, in particular, to have been sceptical about its contribution and cost-effectiveness (Hayes, 2000; Ferriman, 2005). Caller satisfaction with the service has consistently been found to be high with over $90 \%$ of callers reporting that they found the advice very or quite helpful (O'Caithan et al., 2000; Audit Commission, 2002) and most $(85 \%)$ reporting that they had followed the advice given (O'Caithan et al., 2000).

Whether or not users found the service helpful is clearly an important consideration. However safety and effectiveness are also crucial issues which, to date, have received limited investigation. Data from the Audit Commission indicates that adverse events resulting from advice given by NHS Direct are extremely rare (Audit Commission, 2002). However such measures are only a crude indication of effectiveness and the quality of a health service should, perhaps, be judged on the basis of more specific criteria, including patient outcomes. Pearce and Rosen (2000), who conducted an interview based study of health professionals and users involved in the implementation of NHS Direct in London, suggested that perhaps the most important issue of quality is the clinical outcome for each patient-nurse interaction.

\section{Impact of NHS Direct on primary care}

The World Health Organisation (1978) defined primary care as 'the first level of contact of individuals, the family, and the community with the national health system.' In the UK, NHS Direct is now a key part of primary care provision. Munro et al. (2000b) examined the impact of NHS Direct, during its first year, on the use of $A \& E$, ambulance and GP co-operative services in three areas of England. Routine activity in the year before, and the year after, the introduction of NHS Direct were collected from the ambulance services, A\&E departments and GP co-operatives in the study areas, and compared. No changes were found in the use of $A \& E$ departments or ambulance services but there was a reduction in the rate of increase in use of GP co-operatives. Munro et al. conclude that although NHS Direct did not, in its first year, reduce pressures on NHS immediate care services, it may have restrained increasing demand on GP out-of-hours services.

Jones and Playforth (2001) also report a beneficial effect by NHS Direct in dealing with increased demand for telephone advice in A\&E. They compared calls to one A\&E department in October 1998 and October 1999 and recorded the number of calls redirected to NHS Direct. The study showed that, although the number of calls to the hospital seeking advice had increased, the number responded to by the $\mathrm{A} \& \mathrm{E}$ department fell by $72.6 \%$ as a large proportion of calls were redirected to NHS Direct. Jones and Playforth conclude that the introduction of NHS Direct allowed them to put in place a mechanism to reduce the number of calls for advice being dealt with by the department, saving the department's time and resources.

\section{Appropriateness of referrals}

The above studies suggest that NHS Direct has been useful in assisting other primary care services in dealing with increasing demand, which was one of the main driving forces for its introduction (DOH, 2002). However there has been little research assessing whether it has been effective in reducing unnecessary demands on other services. The meaning of 'appropriate' health care has been the subject of much debate (Hopkins, 1993). Professional, patient and societal values all influence judgements about what is deemed to be appropriate. A central issue is the benefit that arises from the referral, compared to the negative consequences that might ensue should a necessary referral not be made. A study by Gaffney et al. (2001) found no significant differences between the triage categories assigned to patients who had been referred to NHS Direct via 999 ambulance 
with those who arrived in A\&E having dialled an ambulance themselves. Gaffney et al. suggest that severity of illness is equally well assessed by self as it is by NHS Direct. The study also found that those who were referred by NHS Direct were less likely to be admitted to hospital or, if discharged, to receive an outpatient appointment than those who had self-dialled. However, there were considerable differences in case mix in the two groups studied which, the authors' state, almost certainly explain the differences in outcome. In order to make more useful comparisons, further work is needed to examine the outcomes of patients who present, either independently or as a referral by NHS Direct, with similar conditions. Gaffney's study compared admission and follow up for those referred by NHS Direct and those who self-dialled. However other indicators of the necessity of the referral, such as the investigations and treatment received, also merit consideration.

\section{Concordance}

The issue of impact on service use and patient outcome are important in assessing the contribution of NHS Direct to quality of care. However, another significant factor is whether callers choose to follow the advice they are given. Foster et al. (2003) examined whether callers followed the advice of NHS Direct advice to attend an A\&E department by comparing NHS Direct data on those referred to $\mathrm{A} \& \mathrm{E}$ with $\mathrm{A} \& \mathrm{E}$ data on actual attendances. They found that just less than two-thirds of callers triaged to A\&E by NHS Direct attended with the same presenting complaint. Although some callers may have attended an A\&E outside of the area studied, up to a third of callers did not follow the advice of NHS Direct to attend A\&E.

Foster et al.'s study relied on data from patient records so provides no insight into the reasons why callers did not follow the advice they were given by NHS Direct. Within health care, the term 'compliance' has been used for many years to describe whether patients do as doctors or nurses advise them. However, 'compliance' suggests that a willingness to follow the wishes of another is desirable (Buchmann, 1997). It is likely that the callers in Foster et al.'s study had reasons for choosing not to do as advised. The term 'concordance' has been used to describe the process of partnership which is central to an effective therapeutic alliance. The concept of concordance is useful in considering, whether, and to what extent, callers choose to follow the advice given by NHS Direct. Young (2004) reviewed the literature about illness behaviour and concluded that individuals' decisions to use a health service and follow advice are related to their perceptions of illness and views of health services. More work is needed to explore, qualitatively, the reasons why people choose to call NHS Direct, their experiences of using the service and the factors that influence their decision whether or not to follow the advice given.

The review of the literature has revealed a lack of empirical evidence relating to the impact on NHS Direct on service use and patient outcome. There is a need for further research to examine the extent to which callers follow advice given by NHS Direct and assess the appropriateness of referrals made by NHS Direct by investigation of clinical outcomes. This paper reports on a study that aimed to examine the subsequent use of health services by callers to NHS Direct and identify the interventions they received.

\section{Aims}

The overall aim of the study was to explore whether callers chose to follow the advice given by NHS Direct to self-care or contact another health service and to examine the interventions they received.

\section{Objectives}

- To compare NHS Direct callers recommended and actual service use.

- To examine the interventions (investigations and treatment) received by NHS Direct callers from other health services.

\section{Method}

\section{Design}

The research formed part of a wider study comparing service use and outcomes of callers to NHS Direct with similar patients attending either a GP or A\&E department. This paper reports on the first part of the study which examined the services used, and interventions received, by callers to NHS Direct. 


\section{Procedure}

Data collection was undertaken via a postal survey of callers who had contacted NHS Direct between June 2004 and January 2005. The study included patients with one of two conditions: (1) abdominal pain and (2) cough and/or sore throat. Focusing on just two conditions was necessary to enable comparison of patient outcomes to be made with similar patients who had contacted either a GP or an A\&E department at a later stage of the study. The two conditions were selected as internal audit data from the participating organizations showed they were common reasons for contacting NHS Direct, or one of the other services. They also represented one normally relatively minor (cough and/or sore throat) and one, at least potentially, more serious condition (abdominal pain).

On four, approximately two monthly occasions, over the eight month period of data collection period all callers who had contacted NHS Direct in the previous three weeks, and met the inclusion criteria, were identified. These callers were sent a letter from NHS Direct explaining the study, together with a consent form and questionnaire.

\section{Inclusion criteria}

Patients who complained of cough and/or sore throat.

Patients who complained of abdominal pain.

Carers/parents whose children complained of either one of the above conditions.

\section{Exclusion criteria}

People with known malignant disease.

Children aged less than one year.

Children aged 12-18 years who called about themselves.

The problem of recall can affect the reliability of questionnaires which ask respondents to remember events which occurred in the past (Fowler, 1993). However it was important that callers would be able to report events related to their illness which occurred after their contact with NHS Direct. Sending questionnaires to those who had called NHS Direct in the three weeks prior to each data collection episode allowed information on patient outcomes to be collected for two weeks after their contact while minimizing the problem of recall.

Primary Health Care Research and Development 2007; 8: 91-100

\section{Sample}

A total of 1019 questionnaires were distributed and 278 were returned. A number of recommended steps (Edwards et al., 2002) were taken to enhance the response rate, including writing to a named individual, inclusion of an explanatory letter from NHS Direct and enclosure of a translation slip to encourage responses from non-English speakers. The response rate of approximately $30 \%$ was therefore disappointing. Response rates to postal questionnaires are frequently low (Bowling, 2002). The potential problem is that of response bias; that those who respond may be different to those who choose not to do so (Edwards et al.,2002). However, Cull et al. (2005) point out, while there are more theoretical opportunities for bias when response rates are low, there is no necessary relationship between response rate and bias. They argue that attention should be devoted to assessments of response bias, rather than relying on response rates as a proxy measure of this. Fife-Shaw (1995) also argues that lower response rates do not mean that the data are without value and stresses the importance of describing the achieved sample as accurately as possible. Comparisons between non-responders and responders can also be used to check for bias.

When it became apparent, during data collection for the present study, that the response rate was likely to be low, demographic data was collected for all callers who were subsequently sent a questionnaire $(n=186)$. Chi-square was used to test for demographic differences between responders $(n=53)$ and non-responders $(n=133)$. No significant differences were found in relation to the variables of condition $\left(\chi^{2}=0.12\right.$, d.f. $\left.=1, P=0.73\right)$, age $\left(\chi^{2}=9.1\right.$, d.f. $\left.=4, P=0.06\right)$ or $\operatorname{sex}\left(\chi^{2}=0.26\right.$, d.f. $=1, P=0.87)$, which suggested that those who responded were not significantly different from those who did not in terms of these characteristics. In addition the relatively large sample size provided a good range of respondents. Details of their characteristics are included within the results.

\section{The questionnaire}

A structured questionnaire was developed consisting of three sections. Section One asked about the caller's condition on the day they contacted NHS Direct and the advice they were given. It also contained sub-sections for callers advised to self-care, callers referred to a GP and callers referred to an 
A\&E department, that asked respondents whether they had followed the advice they were given, how soon they did so and, if they chose not to follow the advice, why they had not done so and what they had done instead. Section Two collected information about what happened to them during their treatment and recovery. Data on investigations received, medication prescribed and admission to hospital, were used as indicators of patient outcome. The final section asked for demographic information about the caller. The questionnaire comprised of 32 questions for callers advised to self-care and 34 for callers referred to a GP or A\&E department. For ease of completion, closed questions were used throughout.

The questionnaire was piloted on a sample of 40 callers to check face and content validity and to test data collection procedures. Fifteen questionnaires were returned, a response rate of $38 \%$. Respondents appeared to understand the questions asked and have little difficulty completing the questionnaire. Minor changes to the questionnaire were made asking respondents to confirm the nature of their condition and to indicate whether their call was made during normal or out-of-hours.

\section{Ethical issues}

Approval for the study was obtained from the Local Research Ethics Committee (LREC). The main ethical issue was that of ensuring patient confidentiality. Questionnaires were sent to callers by NHS Direct. If the caller chose to participate they were asked to complete the questionnaire and return it to the research team at the University. This ensured that patient data was only disclosed to the researchers by the callers themselves, but this indirect means of contact employed may have contributed to the low response rate. A second issue concerned callers aged less than 18 years who may have contacted the service without informing their parent/carer. In order to ensure confidentiality was maintained, children aged 12-18 years were only included in the study if their parent had called on their behalf.

\section{Data analysis}

SPSS (2001) version 11 was used to calculate frequencies and other descriptive statistics to illuminate callers' responses. Results presented in this paper focus on the pathway of service use and interventions received by NHS Direct callers. Of the 278 questionnaires returned, 10 were unable to be used because they were inadequately completed $(n=4)$ or because the respondent did not meet the inclusion criteria $(n=6)$. Results presented are based on 268 questionnaires.

\section{Results}

\section{Demographic characteristics of callers}

It can be seen from Table 1 that a large proportion of the sample $(67 \%)$ were aged less than 40 years, with a higher proportion of female callers $(60 \%)$ than males $(40 \%)$. Only $6 \%$ of callers were from non-white ethnic backgrounds. These sample characteristics reflect the demographic features of NHS Direct callers locally and nationally. It has been recognized that use of NHS Direct by older people and those from non-white ethnic backgrounds is limited and a large proportion (20\%) of calls are made by parents of young children (McIerney et al., 2000; George, 2002).

\section{Contacting NHS Direct}

Section One of the questionnaire asked about the caller's condition on the day they contacted NHS Direct and why they chose to call the helpline. It can be seen from Table 2 that $77 \%$ of callers

Table 1 Demographic characteristics of callers to NHS Direct

\begin{tabular}{lc}
\hline & $\mathrm{N}(\%)$ \\
\hline Age & \\
$1-18$ years & $96(36)$ \\
$19-39$ years & $82(31)$ \\
$40-59$ years & $44(16.5)$ \\
$60+$ years & $44(16.5)$ \\
Total & $266(100)$ \\
Sex & \\
Male & $108(40)$ \\
Female & $159(60)$ \\
Total & $267(100)$ \\
Ethnicity & \\
White British & $251(94)$ \\
Other & $15(6)$ \\
\hline
\end{tabular}

Note: Total sample size $=268$. Numbers vary slightly as some respondents did not answer all questions. 
Table 2 Severity of problem on day of first contact

\begin{tabular}{lc}
\hline Severity of problem & $n(\%)$ \\
\hline Not very severe & $61(23)$ \\
Severe & $171(64)$ \\
Extremely severe & $36(13)$ \\
Total & $268(100)$ \\
\hline
\end{tabular}

Table 3 Extent to which problem prevented usual activities

\begin{tabular}{lc}
\hline $\begin{array}{l}\text { Extent to which problem } \\
\text { prevented usual activities }\end{array}$ & $n(\%)$ \\
\hline Slightly & $40(15)$ \\
Moderately & $87(33)$ \\
Severely & $139(52)$ \\
Total & $266^{\mathrm{a}}(100)$ \\
\hline
\end{tabular}

aTwo respondents did not answer this question.

described their condition as severe $(n=171,64 \%)$ or extremely severe $(n=36,13 \%)$ on the day they contacted NHS Direct. For a large majority $(85 \%)$, their illness had moderately $(n=87,33 \%)$ or severely $(n=139,52 \%)$ prevented them carrying out their usual activities (see Table 3 ). A large proportion of the calls $(n=202,78 \%)$ had occurred outside of normal hours (between 6 p.m. and 8.30 a.m., or at the weekend). Chi-square was used to determine if there was any difference in severity between those who called during normal hours and those who called out-of-hours. No significant difference was found $\left(\chi^{2}=3.84\right.$, d.f. $\left.=1, P=0.15\right)$.

The call to NHS Direct was, in most cases, the person's first point of contact with a health service $(n=184,70 \%)$. However, 75 (28\%) had previously contacted their GP surgery, four had previously contacted an A\&E department, and one had previously sought advice from a pharmacist. Forty-nine of the seventy-five who reported having previously contacted their GP surgery had called the practice outof-hours and had received a recorded message which suggested contacting NHS Direct as an alternative to contacting the out-of-hours GP service, while seven reported that they had previously seen a GP but their condition had since deteriorated. A further twelve of those who had previously seen a GP, and the four callers who had previously attended an A\&E department, had subsequently called NHS Direct because they had wanted some additional health advice while others had been unable to get an appointment with their GP $(n=3)$ or had been advised by staff at their GP surgery to call NHS Direct $(n=3)$.

\section{Advice given by NHS Direct}

Section One of the questionnaire also asked callers about the advice they were given by NHS Direct and whether they followed that advice. Of the 268 callers to NHS Direct, 69 (26\%) were advised to self-care, 144 callers (54\%) were advised to contact a GP, 51 (19\%) were referred to an A\&E department and four $(1 \%)$ were referred to another service.

Among the 69 callers advised to self-care, 64 $(93 \%)$ reported that they had followed the advice to look after themselves at home, while five (7\%) reported that they had chosen not to do so. Of the five, three said they had decided to go to their GP because, despite the advice of NHS Direct, they thought the condition was sufficiently severe to require such a visit. A further two said that their condition deteriorated in the time after their call to NHS Direct, so they then decided to contact their GP.

Of the 144 callers who were advised to contact a GP, $133(92 \%)$ reported that they had done so while $11(8 \%)$ had chosen not to. The reasons given for choosing not to contact a GP were that their condition improved after the call $(n=4)$, the self-care advice given to them by NHS Direct had enabled them to look after themselves at home $(n=3)$, that it was inconvenient to go $(n=2)$ or that they had decided to go to an A\&E department or a chemist instead $(n=2)$.

Of the 51 callers advised to attend an $\mathrm{A} \& \mathrm{E}$ department, $49(96 \%)$ reported that they had done so. Of the two (4\%) who said they did not follow the advice to attend an A\&E department, one reported that she didn't think she was ill enough to do so and instead had contacted her GP, while the other explained that she had waited to see if her condition improved and had subsequently chosen not to attend. Four callers had been referred to another service such as outpatients or an obstetric delivery suite and each of these had followed the advice given.

\section{Interventions received}

Section Two of the questionnaire was concerned with the investigations and treatment received and asked callers about what happened to them during their treatment and recovery. Of the 268 NHS 
Table 4 Interventions received by NHS Direct callers

\begin{tabular}{|c|c|c|c|c|}
\hline Interventions & $\begin{array}{l}\text { Self-care } \\
n=69\end{array}$ & $\begin{array}{l}\text { Referred to } \\
\text { A\&E } \\
n=51\end{array}$ & $\begin{array}{l}\text { Referred to GP } \\
n=144\end{array}$ & $\begin{array}{l}\text { Referred to } \\
\text { other service } \\
n=4\end{array}$ \\
\hline \multicolumn{5}{|l|}{ Investigation } \\
\hline Abdominal pain & 3 & 33 & 37 & 3 \\
\hline Cough/sore throat & 4 & 3 & 15 & 0 \\
\hline Total & 7 & 36 & 52 & 3 \\
\hline \multicolumn{5}{|l|}{ Prescription } \\
\hline Abdominal pain & 3 & 27 & 40 & 2 \\
\hline Cough/sore throat & 15 & 12 & 48 & 1 \\
\hline Total & 18 & 39 & 88 & 3 \\
\hline \multicolumn{5}{|l|}{ Antibiotic } \\
\hline Abdominal pain & 1 & 8 & 18 & 0 \\
\hline Cough/sore throat & 13 & 9 & 43 & 0 \\
\hline Total & 14 & 17 & 61 & 0 \\
\hline \multicolumn{5}{|l|}{ Admission } \\
\hline Abdominal pain & 0 & 18 & 20 & 1 \\
\hline Cough/sore throat & 1 & 2 & 1 & 0 \\
\hline Total & 1 & 20 & 21 & 1 \\
\hline
\end{tabular}

Direct callers, 195 were referred to an A\&E department or told to contact a GP.

Table 4 shows that most callers to NHS Direct who were referred to $A \& E$ or to a GP received one or more of the following interventions; an investigation, a prescription or admission to hospital.

\section{Callers referred to an $A \& E$ Department}

Of the 51 callers referred to an A\&E department, $49(96 \%)$ received one or more interventions, with $36(71 \%)$ receiving an investigation (such as X-Ray, scan or blood tests), 39 (76\%) receiving a prescription and $20(39 \%)$ being admitted to hospital. Of the callers advised to attend A\&E, 39 had called with abdominal pain. Of these callers, 27 (69\%) were given prescriptions which were mainly for antibiotics, painkillers, anti-inflammatory drugs or laxatives. Thirteen of the callers advised to attend an A\&E department had called with cough and/or sore throat. Of these thirteen callers, twelve (92\%) were given a prescription, with nine (69\%) receiving a prescription for antibiotics and two were admitted to hospital.

\section{Callers advised to contact a GP}

Of the 144 callers referred to a GP, $106(74 \%)$ received one or more interventions, with $52(36 \%)$ receiving an investigation (such as an X-Ray, scan or blood test), $88(61 \%)$ receiving a prescription and $21(15 \%)$ being admitted to hospital. Of the callers advised to contact a GP, 81 had called with abdominal pain. Of these callers, 40 (49\%) were given prescriptions, which again were mainly prescriptions for antibiotics, painkillers, antiinflammatory drugs or laxatives. Twenty $(25 \%)$ of those with abdominal pain who were referred by NHS Direct to a GP were admitted to hospital. Sixty-three of the callers advised to contact a GP had complained of cough and/or sore throat. Of these callers, 48 (76\%) received a prescription, with $43(68 \%)$ being given a prescription for antibiotics. One caller with cough and/or sore throat who was advised to contact a GP was admitted to hospital.

\section{Callers advised to self-care}

Of the 69 callers advised by NHS Direct to selfcare, $47(68 \%)$ reported that they did not receive any further intervention. Eighteen callers (26\%) who were advised to self-care, reported they had been given a prescription for medication. Of these, five reported that they had chosen not to follow the advice to self-care either, because they thought the condition was sufficiently severe to require a visit to a GP $(n=3)$, or because the condition had deteriorated after their call $(n=2)$. Thirteen callers who said that they had followed the advice to self-care, also reported that they had afterwards visited their GP about their health problem. Twelve of these had called NHS Direct with cough and/or sore throat. Of these, 11 were given a prescription 
for antibiotics by the GP, one a prescription for a painkiller and one an unspecified prescription. The other had called with abdominal pain and had received a prescription for an antacid from the GP. It was evident, therefore, that all 18 NHS Direct callers who had been advised to self-care and, yet, had received a prescription, had additionally chosen to contact a GP.

\section{Discussion}

This study has investigated the impact of NHS Direct on use of health services, and the investigations and treatment subsequently received, by callers to NHS Direct in one part of the UK. Despite a disappointing response rate and reliance on callers' self-reports, the study provides insight into the reasons why people contact NHS Direct, whether they followed the advice given and the health interventions they received.

A primary aim of NHS Direct is to provide easily accessible health advice 24-h a day (Munro et al., $2000 \mathrm{~b})$. The large proportion of callers $(77 \%)$ who described their condition as severe or extremely severe on the day they called, together with the large number $(85 \%)$ who reported their illness had moderately or severely prevented them from carrying out their usual activities, indicates that a considerable majority of callers to NHS Direct saw themselves as having a significant health problem for which they needed health advice and/or information. For most people their call to NHS Direct was their first point of contact with the primary care services and was chosen because of its accessibility and convenience. Those who had contacted NHS Direct when their GP out-of-hours ansaphone message suggested it as an alternative to contacting an emergency doctor, said they had called the helpline because they did not think their problem was an emergency, but they did need some health advice.

A second aim of NHS Direct is to help improve the quality, increase cost-effectiveness and reduce unnecessary demands on other services (Munro et al., 2000). In the present study over $90 \%$ of respondents reported they had followed the advice to self-care or contact another service, a result that is comparable with other NHS Direct follow-up surveys (Audit Commission, 2002). The present study also examined the investigations and treatment received by NHS Direct callers as an indicator of the necessity of the referral and found that the majority of callers who were referred to an A\&E department or a GP received one or more interventions which appeared to justify the referral.

Of those referred to an A\&E department or a GP by NHS Direct, $65 \%$ received a prescription. Cox and Jones (2001) report that, prior to an intervention to decrease prescribing rates for antibiotics, the rate in their UK practice for patients with sore throat was $56 \%$. In the present study $67 \%$ of the callers referred to an A\&E department or a GP with cough and/or sore throat received a prescription for antibiotics. Social factors and individual practitioners' prescribing habits can have an impact on prescription rates (Sutter et al., 2001). However the high rate of prescriptions, and specifically prescriptions for antibiotics for those with cough and/or sore throat, in the present study suggests that the prescription rates for patients referred by NHS Direct were somewhat higher than usual prescribing practice. A large proportion of the NHS Direct callers received a prescription which was specific to their condition, with most of those with abdominal pain receiving a prescription for antibiotics, painkillers, anti-inflammatory drugs or laxatives and those with cough and/or sore throat receiving a prescription for antibiotics.

Of callers referred to A\&E, 20 (39\%) were admitted to hospital, with 18 of the 38 (47\%) callers with abdominal pain being admitted. At the time of data collection (2004), admission rates of patients from A\&E to hospital, nationally, were 16-17\% (DOH, 2005). Locally, rates were slightly higher for three of the hospitals included in the study (16-22\%) although one had an admission rate of $37 \%$. Admission rates for the two included conditions may be different from those in general. However, the fact that about $40 \%$ of those referred by NHS Direct to an A\&E department, and, of those, nearly $50 \%$ of those with abdominal pain, were admitted to hospital, lends support to the necessity of the referrals.

Although in most cases, callers referred to another health service by NHS Direct received one or more interventions which appeared to support the need for the referral, this was not true in all cases. Whilst only $4 \%$ of those referred to an A\&E department received no further intervention, about a quarter (26\%) of those referred to a GP did not receive either an investigation, a prescription or admission to hospital. Determining 
the nature and severity of a health problem such as abdominal pain or cough and/or sore throat is difficult. In addition, there are certain circumstances where NHS Direct may refer a caller to the GP when a further intervention may not be expected. For example the Royal College of Surgeons currently recommends that patients with localized abdominal pain receive a face to face consultation. However, the fact that $26 \%$ of callers advised by NHS Direct to contact a GP received no further intervention appears to confirm the comment of the Audit Commission (2002) that advice from NHS Direct tends to err on the side of caution.

There was also some duplication of service use among those whom NHS Direct advised to selfcare. In the present study, $26 \%$ of those who were advised to self-care chose afterwards to contact a GP about their condition. This indicates that people are contacting two, or more, services for the same health problem. With the rise of NHS Direct, Walkin Centres (Salisbury et al., 2002) and other innovative services, there is increasing complexity in primary care services. While this offers greater choice, unnecessary duplication of service use and increasing fragmentation of care may result. Health professionals need to be aware that patients might have previously contacted another health service and, as well as making sure patients are aware of the range of services which are available to them, may need to provide advice on effective use of services.

NHS Direct was established in the UK to provide 24 -h confidential health advice and information to callers. Issues related to patient outcomes and service effectiveness are important concerns for callers, health professionals and NHS Direct itself. Yet, the service may also have a benefit to callers that is distinct from the outcome of care, or whether the individual chooses to follow the advice given. Coulter (2005) suggests that some of the things patients want most from primary care are fast access to reliable health advice and easier, more flexible access to services. Most patients are happy to consult with a nurse, or other health professional, when it is appropriate to do so (Lattimer et al., 1998) and use of alternative ways of accessing health advice and information are becoming popular (Ellins and Coulter, 2005). Nursing has long emphasized the central importance of a holistic approach to care, which recognizes patients' emotional, as well as their physical needs
(Smith, 1992). The high levels of satisfaction found with NHS Direct (Munro, 2002) suggests that this telephone based helpline, staffed by nurses, is valued by callers as an important source of primary care.

\section{Limitations}

This was a descriptive survey of NHS Direct callers with cough and/or sore throat and abdominal pain. A limitation of the study was the poor response rate. Although no significant difference was found in the demographic background of responders and non-responders, we must be cautious about assuming the responses are obtained are typical of other NHS Direct callers with these conditions. The results from the statistical limb of the study also require cautious interpretation. Power as a function of sample size was not calculated and the results are based on face inferences, based on proportions. Therefore the precision, reliability and generalizability of the results have not been verified.

\section{Conclusions}

This study has examined the use of NHS Direct by callers with cough and/or sore throat or abdominal pain, whether they followed the advice they were given by NHS Direct to self-care or contact another service and the interventions they subsequently received. The study found that most of those advised to self-care did not require any further intervention, although a minority did afterwards attend their GP and receive a prescription. Almost all callers to NHS Direct who were referred to A\&E or a GP received a further intervention which appeared to justify the referral. Further analysis is currently being undertaken to examine whether differences exist in patient outcomes for callers who are referred to an $A \& E$ department or GP by NHS Direct and patients who attend directly. In addition, we are also undertaking analysis of qualitative interviews with callers which explored their perceptions of the quality of care they received and the factors that influenced them to follow, or not, the advice given by NHS Direct. 


\section{References}

Audit Commission. 2002: NHS Direct in England. Report of the Controller and Auditor General. HC 505. London: HMSO.

Bowling, A. 2002: Research Methods in Health: Investigating Health and Health Services, second edition. Buckingham: Open University Press.

Buchmann, W.F. 1997: Adherence: a matter of self-efficacy and power. Journal of Advanced Nursing 26, 132-37.

Bunn, F., Byrne, G. and Kendall, S. 2005: The effects of telephone consultation and triage on healthcare use and patient satisfaction: a systematic review. British Journal of General Practice 55, 956-61.

Christensen, M. and Olensen, F. 1998: Out of hours service in Denmark: evaluation five years after reform. British Medical Journal 316, 1502-505.

Coulter, A. 2005: What do patients and the public want from primary care? British Medical Journal 331, 1199-201.

Cox, C. and Jones, M. 2001: Is it possible to decrease antibiotic prescribing in primary care? An analysis of outcomes in the management of patients with sore throats. Family Practice $18,9-13$

Cull, W.L., O'Connor, K.G., Sharp, S. and Tang, S.S. 2005: Response Rates and Response Bias for 50 Surveys of Paediatricians. Health Services Research 40, 213-26.

Department of Health. 2002: The NHS Plan. London: Department of Health.

Department of Health. 2005: Accident and emergency: attendances, admissions and total time spent. Retrieved from http://www.performance.doh.gov.uk/hospitalactivity/ data requests/index.htm

Edwards, P., Roberts, I., Clarke, M., Di Guiseppi, C., Pratap, S., Rentz, R. and Kwan, I. 2002: Increasing response rates to postal questionnaires: systematic review. British Medical Journal 324, 1183-86.

Ellins, J. and Coulter, A. 2005: How Engaged are People in Their Health Care? London: Health Foundation.

Ferriman, A. 2005: BMA Annual Representative Meeting, Manchester, 27-30 June: NHS Direct and NHS 24 condemned as flawed by BMA conference. British Medical Journal 331, News Extra.

Fife-Schaw, C. 1995: Surveys and sampling issues. In Breakwell, G.M., Hammond, S. and Fife-Schaw, C. editors, Research Methods in Psychology. London: Sage.

Foster, J., Jessop, L. and Chakraborti, S. 2003: Do callers to NHS Direct follow the advice to attend an accident and emergency department. Emergency Medicine Journal 20, 285-88.

Fowler, F.J. 1993: Survey Research Methods, second edition. London: Sage.

Gaffney, P., Crane, S., Johnson, G. and Playforth, M. 2001: An analysis of calls referred to the emergency 999 service by NHS Direct. Emergency Medicine Journal 18, 302-304.
George, S. 2002: NHS Direct Audited (Editorial). British Medical Journal, 324, 558-559.

Hanlon, G., Strangleman, T., Goode, J., Luff, D., O’Caithan, A. and Greatbatch, D. 2005: Knowledge, technology and nursing: the case of NHS Direct. Human Relations 58, 147-71.

Hayes, D. 2000: The case against NHS Direct. Doctor 13,36-39.

Hopkins, A. 1993: What do we mean by appropriate health care? Report of a working group prepared for the Director of Research and Development or the NHS Management Executive. Quality in Health Care 2, 117-23.

Jones, J. and Playforth, M.J. 2001: The effect of the introduction of NHS Direct on requests for telephone advice from an accident and emergency department. Emergency Medicine Journal 18, 300-301.

Lattimer, V., George, S. and Thompson, F. 1998: Safety and effectiveness of nurse telephone consultation in out-ofhours primary care. British Medical Journal 317, 1054-59.

McIerney, J., Chillala, S., Read, C. and Evans, A. 2000: Target communities show poor awareness of NHS Direct (letter). British Medical Journal 321, 1077.

Munro, J. 2002: Ringing endorsement. Nursing Times 98, 10-11.

Munro, J. Nicholl, J., O'Caithan, A. and Knowles, E. 2000a: Evaluation of NHS Direct First Wave Sites Second Interim Report to the Department of Health. The Medical Research Unit, University of Sheffield.

Munro, J. Nicholl, J., O'Caithan, A. and Knowles, E. 2000b: Impact of NHS Direct on demand for immediate care: observational study. British Medical Journal 321, 150-53.

O'Caithan, A., Munro, J.F., Nicholl, J.P. and Knowles, E. 2000: How helpful is NHS Direct? Postal survey of callers. British Medical Journal 320, 1035.

Pearce, K. and Rosen, R. 2000: NHS Direct: Learning from the London Experience. London: Kings Fund.

Salisbury, C. et al. 2002: What is the role of walk-in centres in the NHS. British Medical Journal 324, 399-402.

Smith, P. 1992: The Emotional Labour of Nursing: Its Impact on Interpersonal Relations, Management and the Educational Environment in Nursing. London: Macmillan.

Sprivulis, P., Carey, M., and Rouse, I. 2004: Compliance with advice and appropriateness of emergency presentation following contact with HealthDirect telephone triage service. Emergency Medicine, Australia 16, 35-40.

SPSS Inc. 2001: Version 11, Statistical Package for the Social Sciences. Chicago, IL: SPSS Inc.

De Sutter Al, et al. 2001: Antibiotic prescribing in acute infections of the nose or sinuses: a matter of personal habit. Family Practice 18, 209-13.

World Health Organisation. 1978: Declaration of Alma-Ata. World Health Organisation.

Young, J.T. 2004: Illness behaviour: a selective review and synthesis. Sociology of Health and Illness 26, 1-24. 